\title{
The Teaching Level Evaluation of Foreign Teachers Based on Analytic Hierarchy Process Method
}

\author{
Zhang Haolong, Xu Jin \\ Jining Polytechnic, Jining, Shandong Province 370800, China
}

Keywords: analytic hierarchy process; foreign teachers in universities; teaching level; evaluation method

\begin{abstract}
Teaching evaluation plays the key role in the process of teaching quality in colleges and universities. This measure is helpful to carry on the omni-directional comprehensive evaluation to improve teaching level. The execution shall act on comprehensive, scientific, objective and systematic indicators. At present, many colleges and universities have hired foreign teachers. Comparatively speaking, the teaching level of foreign teachers at current makes a more significance and the related teaching evaluation is urgently needed to be improved. For foreign teachers in colleges and universities if analytic hierarchy process can be used to complete the teaching evaluation, then more objective evaluation conclusion will be made, and the present situation is helpful to improve comprehensive foreign teachers' teaching level.
\end{abstract}

\section{Introduction}

In recent years, the new educational reform is being gradually executed, and the corresponding process has gained comprehensive in-depth. Analytic Hierarchy Process can also be abbreviated as AHP-- this kind of evaluation method with structural and comprehensive characteristics, and all indicators involved also has been comprehensively improved. From the current situation, in view of the foreign teachers usually use qualitative evaluation as the core of evaluation mode, but such evaluation overall lack of comprehensiveness, therefore it needs to be overall perfected and improved [1]. Evaluation index based on analytic hierarchy process (AHP) are more scientific, enhancing the role of weight index. So we can draw a conclusion that, analytic hierarchy process (AHP) used in the present stage of teaching evaluation measures will eliminate blind spots, conforming to basic requirements of new educational reform in the field of teaching evaluation.

\section{The Important Significance of Teaching Evaluation}

Teaching level evaluation refers to employing specific methods and measures to carry out classroom evaluation--aims and objectives of evaluation is to improve the level of comprehensive teaching. Facing the situation of new curriculum reform, teaching evaluation should be established under the premise of that, for all teaching process and teaching links need to carry out comprehensive evaluation. Teaching evaluation, therefore, should be fair and objective, ensuring that the quality of teaching within the maximum limit. From this perspective, the teaching evaluation is not limited to the evaluation itself, but constitutes the important way of promoting the teaching level [2]. In particular, the important value of teaching evaluation is as follows:

First of all, teaching evaluation is helpful to arose enthusiasm and to improve comprehensive teaching level. In essence, the teaching evaluation should focus on teaching links of different subjects, and each course should carry out real-time classroom evaluation. So you can know, optimizing and improving the quality of teaching evaluation is helpful to enlighten thinking, and this is due to the teaching evaluation usually involve defects in the classroom, and then the teacher can clearly improve the basic direction. Once the quality of teaching was comprehensively improved, the enthusiasm of students has also promoted [3].

Secondly, teaching evaluation and the overall teaching quality is an integral inner link. From the 
perspective of teaching evaluation, students should play the dominant role in the class. A comprehensive class assessment can ensure aims and objectives in accordance with their aptitude, at the same time, the classroom instruction of each process was optimized. On the basis of comprehensive introspection, holistic improvement of present stage of every classroom can be conducted, thus it is advantageous to the comprehensive optimization of each link of teaching.

Thirdly, conducting scientific evaluation will help to cultivate excellent teachers, optimizing teacher management. This is because the teaching evaluation usually involves the basic structure of teaching staff, and it fully proves their defects, and then gives highly targeted improvement measures [4]. From the point of administrators, school managers should also be closely combined with the evaluation conclusion during different period to optimize the whole faculty. Thus, teaching evaluation should be able to keep the scientific nature to ensure they get stronger reliable and objective conclusion.

\section{Aims and Principles of Teaching Evaluation}

Teaching evaluation should reflect the comprehensive teaching level of foreign teachers, so it is necessary to pay more attention to it. Foreign teachers have their own particularity, therefore it is needed to combining the basic characteristics of foreign teachers when evaluating, and it needs to comply with specific evaluation criteria. So in the light of the concrete in foreign teachers to carry out the evaluation, it is necessary in accordance with the principle of the evaluation as the following:

Firstly, it should be practical and scientific. From the perspective of teaching practice, it is necessary to set up a multi-level evaluation index for foreign teachers in colleges and universities to ensure that all indexes are more scientific. When setting on indicators, it shall keep clear logical and clear structure; try to avoid tedious design of evaluation index. Only to ensure that the practical and scientific teaching evaluation will be much more practical, thus it can obtain the quantitative and qualitative comprehensive conclusions [5]. On the basis of the scientific aim to set up the evaluation indexes to ensure them comply with diversity classification criteria. This is because evaluation indexes contain different content, and overlaps need to be avoided.

Secondly, it should be good-oriented. The aim of the teaching evaluation is guiding the daily teaching, especially foreign teachers. Thus, teaching evaluation should be with rather strong guidance. Orientation refers to means of teaching evaluation, making foreign teachers realize their advantages and shortcomings in the daily teaching, and thus make it available for operation improvement measures. Teaching evaluation should be able to get strong guidance evaluation information, directing daily teaching.

Thirdly, it should be easy-operated. Operability means feasibility-- the evaluation conclusion should be instructive and practical and it will provide comprehensive guidance in daily teaching. Simple evaluation indicators are employed to comprehensively reflect advantages and disadvantages of foreign teachers' daily teaching. Teaching evaluation is executed in a simple and convenient way to ensure the streamline of organization from the beginning, avoiding the waste of financial resources and manpower.

Fourthly, it should be diversified. From the perspective of diversity, it should be embodied in various evaluation modes. In most cases, if only test mode or measuring method is used, more precious evaluation conclusion is so difficult to get [6]. Thus it can be seen that evaluation method should be diversified and every indicator involved in daily teaching need to be comprehensively measured. Specifically speaking, teaching evaluation usually involves many links--investigation, questionnaire, and observation records and so on, on the basis of that, closely combining over behavior and psychological states and paying more attention to individual differences.

\section{Specific Evaluation Methodology}

(I) The characteristic and contents of analytic hierarchy process

Analytic hierarchy process should be established on the basis of multi-level 
relations--comprehensive evaluation will be made on all levels involved in daily teaching. Specifically, analytic hierarchy process (AHP) ought to divide class teaching into different levels-- destination layer, scheme layer and criterion layer, and then corresponding weight and evaluation index will be achieved after compared term by term. So we can draw a conclusion that, analytic hierarchy process (AHP) is to build more intuitionistic evaluation index, hereby, it can reflect its important position in the whole system. When operating, we need to resolve multifarious problems, building layering hierarchical evaluation mode through analysis and abstraction. Among these, the upper element should be able to control the lower element.

(II) Partitioning model levels

It is necessary to construct model if you employ the analytic hierarchy process (AHP) in the teaching evaluation. The premise of constructing mode is dividing different levels. In general, when evaluating foreign teachers' teaching effectiveness, the whole model can be classified into highest, middle and bottom layers [7]. Among them, the target layer is the highest layer, including unique elements and representing the ideal state of classroom efficiency; each link in the middle layer should involve each link of the class teaching, and the bottom layer is available to perform optimized scheme, which involves feasible measures of optimizing classroom teaching.

(III)Building matrix and draw conclusion

For all evaluation indexes, complete matrix is made after contrast evaluation. In particular, in the process of constructing matrix different links need to be specialized, such as teaching attitude, teaching methods, teaching preparation, teaching guidance and assessment, etc. By using consistency check method, you can get the corresponding weights of indexes at all levels. After comprehensive evaluation, foreign teachers can understand their gaps of teaching, and then improve abilities with appropriate measures. Therefore, perfecting evaluation index is helpful to obtain more precious classroom evaluation conclusion.

\section{Conclusion}

In essence, with the aid of the fuzzy evaluation measures and means, the analytic hierarchy process builds a more comprehensive teaching evaluation system. Foreign teachers play significant role in daily teaching in universities and at the same time, they take up a special position. In order to improve foreign teachers' teaching level, it is necessary to implement new teaching evaluation on the current situation, and analytic hierarchy process (AHP) model is the typical way. However, it should not be ignored that analytic hierarchy process (AHP) used in the new teaching evaluation also shows disadvantages and defects, this is because analytic hierarchy process (AHP) is so stable, thus it is not good for flexible evaluation. in the following practice, people need to make constant exploration to ensure the incorporation of teaching evaluation and analytic hierarchy process (AHP), highlighting the unique advantages of AHP on the basis of that.

\section{References}

[1] Liu Wei, Jin Lin. The Evaluation Teaching Level of Teachers in Colleges and Universities Based on Analytic Hierarchy Process [J]. Statistics and Decision, statistics and decision, 2016(23):58-61.

[2]Wan Laihong, Sun Jingbo. The Construction and Practice of Ability \& Quality Evaluation System of P. E teachers in Universities [J]. Journal of Wuhan Institute of Physical Education, 2016(11):87-91+100.

[3]Wang Xin. Evaluation Of Teacher's Teaching Level Based On Rough Set And Analytic Hierarchy Process [J]. Microcomputer Applications,2016(04):42-44.

[4] Wu Qingtian, Liu Tao. The Analysis of the Comprehensive Evaluation of Teaching Quality of Teachers in Colleges and Universities-- The Uncertainty of the Multicriterion Group Decision Method Based on Cloud Model [J]. The Research of Macroscopical Quality,2014(04):114-122.

[5]Wang Jiancai, Fu Ming. The Study of Teaching Quality Evaluation Based on Cloud Model and 
Analytic Hierarchy Process [J]. Value Engineering,2013(23):290-291.

[6]Yang Wenmin. The Study on Evaluation Index System for Teaching Ability of Physical Education Teachers in Colleges and Universities [J]. Zhejiang Sport Science,2013(03):82-85.

[7]Yang Zhen, Mu Zhanjun. The Teaching Quality Evaluation in Colleges and Universities Based on Analytic Hierarchy Process[J]. Journal of Inner Mongol University of Technology(Social Science Edition),2012(01):111-115+122. 